\title{
Metric versus observable operator representation, higher spin models
}

\author{
Andreas Fring a and Thomas Frith ${ }^{\mathrm{b}}$ \\ Department of Mathematics, City, University of London, Northampton Square, London EC1V 0HB, UK
}

Received: 14 September 2017 / Revised: 15 January 2018

Published online: 15 February 2018

(C) The Author(s) 2018. This article is published with open access at Springerlink.com

\begin{abstract}
We elaborate further on the metric representation that is obtained by transferring the timedependence from a Hermitian Hamiltonian to the metric operator in a related non-Hermitian system. We provide further insight into the procedure on how to employ the time-dependent Dyson relation and the quasi-Hermiticity relation to solve time-dependent Hermitian Hamiltonian systems. By solving both equations separately we argue here that it is in general easier to solve the former. We solve the mutually related time-dependent Schrödinger equation for a Hermitian and non-Hermitian spin 1/2,1 and $3 / 2$ model with time-independent and time-dependent metric, respectively. In all models the overdetermined coupled system of equations for the Dyson map can be decoupled algebraic manipulations and reduces to simple linear differential equations and an equation that can be converted into the non-linear Ermakov-Pinney equation.
\end{abstract}

\section{Introduction}

Standard quantum mechanics allows for many equivalent variants to describe the same physical observables. The well-known reason for this is that expectation values are computed from ambiguous quantities in which the individual components can be modified while the overall expression for the expectation values are left unchanged. Gauge transformations are prominent examples for such possible alterations. For time-dependent situations the well-known equivalence between the Schrödinger and the Heisenberg picture allows to change from time-dependent states and time-independent operators to time-independent states and time-dependent operators, respectively. Recently we [1] argued that in time-dependent-symmetric/quasi-Hermitian systems [2-4] another variant is possible in which the timedependence is transferred from observables to metric operators. We will refer to the former as the observable operator representation and the latter as the metric representation by indicating the time-dependent object in the name of the representation. These physically equivalent representations are made possible in this setting as it always involves nontrivial metric operators on the non-Hermitian side. For Hermitian systems one can employ time-dependent Darboux transformation to map time-dependent Hamiltonian systems to time-independent ones [5, 6].

In [1] we demonstrated that the time-dependent Schrödinger equation (TDSE) for a time-dependent Hermitian Hamiltonian, $h(t)=h^{\dagger}(t)$, and the easier TDSE for a time-independent Hermitian Hamiltonian, $H \neq H^{\dagger}$,

$$
h(t) \phi(t)=i \hbar \partial_{t} \phi(t) \text { and } H \Psi(t)=i \hbar \partial_{t} \Psi(t),
$$

may be treated equivalently. In the proposed scenario the Hermitian system is governed by a time-dependent Hamiltonian $h(t)$ and a standard time-independent metric operator $\mathbb{I}$, i.e. the unit operator, whereas the non-Hermitian system is characterized by the time-independent Hamiltonian $H$ and a non-standard time-dependent metric operator $\rho(t)$. The associated inner products in both systems are equivalent in the sense that

$$
\langle\phi(t) \mid \mathbb{I} \phi(t)\rangle=\langle\Psi(t) \mid \rho(t) \Psi(t)\rangle,
$$

where the two wave functions $\phi(t)$ and $\Psi(t)$, solving the respective equation in $(1)$, are connected by the time-dependent invertible Dyson operator $\eta(t)$ as

$$
\phi(t)=\eta(t) \Psi(t) .
$$

\footnotetext{
a e-mail: a.fring@city.ac.uk

b e-mail: thomas.frith@city.ac.uk
} 
The metric operator in (2) and the Dyson operator in (3) are simply related as $\rho(t):=\eta^{\dagger}(t) \eta(t)$. Thus in this picture the time-dependence has been moved from the Hamiltonian in the Hermitian system to the metric operator in the non-Hermitian system.

There are two central equations that serve to determine the quantities involved in the equations above. The first one, the time-dependent quasi-Hermiticity relation

$$
H^{\dagger} \rho(t)-\rho(t) H=i \hbar \partial_{t} \rho(t)
$$

results by demanding that the time-evolution is unitary, that is the expectation values in (2) are preserved in time. Setting the time derivative of (2) to zero and using the TDSE (1) leads to (4). The second equations, the time-dependent Dyson relation

$$
h(t)=\eta(t) H \eta^{-1}(t)+i \hbar \partial_{t} \eta(t) \eta^{-1}(t)
$$

is obtained by substituting (3) into (1).

It was noted some time ago [7-11] that as a consequence of the Dyson relation (5) the Hamiltonian satisfying the TDSE (1) is not observable ${ }^{1}$, since observables $\mathcal{O}$ in the non-Hermitian system need to be quasi-Hermitian, meaning they have to be related to a corresponding observable o, i.e. a self-adjoint operator, in the Hermitian system as $o(t)=\eta(t) \mathcal{O}(t) \eta^{-1}(t)$. The non-observability is also a feature when the Hamiltonian is explicitly time-dependent, i.e. even for $H \rightarrow H(t)$. Furthermore, this implies that $H$ is not the operator that characterizes the energy but instead the operator

$$
\tilde{H}(t)=\eta^{-1}(t) h(t) \eta(t)=H+i \hbar \eta^{-1}(t) \partial_{t} \eta(t),
$$

that does not satisfy the TDSE, and is therefore by definition not a Hamiltonian, is the energy operator in the non-Hermitian system. The relation between the expectation values in the different systems is easily verified to be

$$
\langle\phi(t) \mid h(t) \phi(t)\rangle=\langle\Psi(t) \mid \rho(t) \tilde{H}(t) \Psi(t)\rangle,
$$

supporting the above statement. As demonstrated in [1] unitary time-evolution operators $u\left(t, t^{\prime}\right)$ and $U\left(t, t^{\prime}\right)$ that evolves a state as $\phi(t)=u\left(t, t^{\prime}\right) \phi\left(t^{\prime}\right)$ or $\Psi(t)=U\left(t, t^{\prime}\right) \Psi\left(t^{\prime}\right)$, respectively, from a time $t^{\prime}$ to $t$ may also be constructed when $\phi(t)$ and $\Psi(t)$ have been obtained. We will not comment here further on non-unitary time-evolution as this can be dealt with easily in a similar fashion to the time-independent scenario by using biorthonormal basis states.

Since eqs. (4) and (5) describe highly overdetermined systems it is a priori not evident whether they possess any solutions at all and if they do whether they are meaningful. Remarkably such solutions do exist and can be found as was demonstrated for time-dependent [11,13] and time-independent Hamiltonians [1]. Here we provide further solutions, focussing on the limitations and in particular on the different solution procedures. In [1] we pursued the following process: Starting from a given a non-Hermitian Hamiltonian $H$ we solved the time-dependent quasi-Hermiticity relation (5) first, which seems most natural as it only involves one unknown quantity, namely $\rho(t)$. Assuming the Dyson operator $\eta(t)$ to be Hermitian, it can in principle be computed from $\rho(t)$ by taking its square root. Subsequently one may compute the Hermitian counterpart $h(t)$ by direct evaluation of the right-hand side of the time-dependent Dyson relation (5). As we will demonstrate in more detail below, taking the square root in this case can be rather awkward and to avoid this step we pursue here a different approach by solving the time-dependent Dyson relation first. As we will see, this is more efficient, but evidently requires some initial guess about the structure of the Hermitian Hamiltonian.

The models we consider here are slightly modified versions of the lattice Yang-Lee model $[14,15]$

$$
H_{N}^{s}=-\frac{1}{2} \sum_{j=1}^{N}\left(c_{y} S_{j}^{y}+\omega c_{\omega} \vec{S}_{j} \cdot \vec{S}_{j+1}+i c_{x} \gamma S_{j}^{x}\right), \quad \omega, \gamma, c_{x}, c_{y}, c_{\omega} \in \mathbb{R},
$$

where we allow for higher spin representations for the matrices $S_{j}^{x}, S_{j}^{y}, S_{j}^{z}$ at site $j$ labelled by $s$. We assume the standard boundary conditions $\vec{S}_{1}=\vec{S}_{N+1}$. Our model parameters are $\omega, \gamma \in \mathbb{R}$ and the constants $c_{x}, c_{y}, c_{\omega}$ are conveniently adjusted for the particular representations. Here we will consider the one-site models and attempt, in analogy to the study in [1], to map the non-Hermitian Hamiltonians to Hermitian Hamiltonians of the form

$$
h(t)=-\frac{1}{2}\left[\omega \mathbb{I}+\chi(t) S_{z}\right]
$$

where initially $\chi(t)$ is an arbitrary unknown function of $t$. It turns out that in all spin models considered the timedependent function $\chi(t)$ is restricted to obey an equation that can be converted easily into the non-linear ErmakovPinney equation.

\footnotetext{
1 This fact cannot be changed by imposing the additional constraint $i \hbar \partial_{t} \eta(t)=\eta(t) H(t)$, as suggested in [12], as this evidently produces a factor 2. Moreover, this constraint implies that the metric has to be time-independent so that $\eta(t)$ must be either non-Hermitian or also time-independent.
} 


\section{A solvable equivalence pair of spin-(1/2) models}

The simplest version of $H_{N}^{s}$ is the one-site spin-(1/2) model. Taking the matrices $S_{j}^{x}, S_{j}^{y}, S_{j}^{z}$ simply to be the standard Pauli spin matrices $\sigma_{j}^{x}, \sigma_{j}^{y}, \sigma_{j}^{z}$ and adjusting the constants $c_{x}=c_{y}=1, c_{\omega}=1 / 3$, the Hamiltonian (8) acquires the form

$$
H_{1}^{1 / 2}=-\frac{1}{2}\left(\sigma_{y}+\frac{\omega}{3} \vec{\sigma} \cdot \vec{\sigma}+i \gamma \sigma_{x}\right)=-\frac{1}{2}\left(\begin{array}{cc}
\omega & i(\gamma-1) \\
i(\gamma+1) & \omega
\end{array}\right) .
$$

The corresponding TDSE (1) is easily solved by

$$
\Psi_{ \pm}(t)=\left(\begin{array}{c} 
\pm i(1-\gamma) \\
\phi
\end{array}\right) e^{-i t E_{ \pm}}, \quad E_{ \pm}=-\frac{\omega}{2} \pm \frac{\phi}{2}
$$

where $\phi:=\sqrt{1-\gamma^{2}}$. Thus, this model exhibits the typical feature for $\mathcal{P} \mathcal{T}$-symmetric/quasi-Hermitian systems [3,4] that despite being described by a non-Hermitian Hamiltonian there exists a range for the model parameters, in this case $|\gamma| \leq 1$, for which the eigenvalue spectrum is real. Next we will solve the time-dependent Dyson relation (5) and the time-dependent quasi-Hermiticity relation (4) in more detail and compare the advantages of one approach over the other.

\subsection{Solutions of the time-dependent quasi-Hermiticity relation}

Assuming the time-dependent metric operator to be Hermitian we take it to be in the most generic form

$$
\rho(t)=\left(\begin{array}{cc}
\rho_{1}(t) & \rho_{2}(t)-i \rho_{3}(t) \\
\rho_{2}(t)+i \rho_{3}(t) & \rho_{4}(t)
\end{array}\right),
$$

with unknown real functions $\rho_{i}, i=1, \ldots, 4$. Substituting this Ansatz into (4) and reading off the real and imaginary parts in each matrix entry yields the four constraining first-order differential equations

$$
\dot{\rho}_{1}=(1+\gamma) \rho_{2}, \quad \dot{\rho}_{2}=\rho_{1} \frac{\gamma-1}{2}+\rho_{4} \frac{\gamma+1}{2}, \quad \dot{\rho}_{3}=0, \quad \dot{\rho}_{4}=(\gamma-1) \rho_{2} .
$$

As common we adopt the convention to indicate derivatives with respect to time by an overdot. The general solution to these equations is easily obtained as

$$
\rho_{1}(t)=\frac{1+\gamma}{\phi} \Gamma_{b_{2}}^{-b_{1}}+b_{4}, \quad \rho_{2}(t)=\Gamma_{b_{1}}^{b_{2}}, \quad \rho_{3}(t)=b_{3}, \quad \rho_{4}(t)=\frac{1-\gamma}{\phi} \Gamma_{-b_{2}}^{b_{1}}+\frac{1-\gamma}{1+\gamma} b_{4},
$$

where we abbreviate $\Gamma_{x}^{y}:=x \sin (\phi t)+y \cos (\phi t)$ and introduced the real integration constants $b_{1}, b_{2}, b_{3}, b_{4}$. To find (14) we just need to solve a harmonic-oscillator equation obtained from computing $\ddot{\rho}_{2}$ and the subsequent use the expressions for $\dot{\rho}_{1}, \dot{\rho}_{4}$. Once $\rho_{2}$ is known the remaining integrals are simply of first order.

In principle, we can take now the square root by diagonalizing $\rho$ first as $\rho=U D U^{-1}$ and subsequently computing $\sqrt{\rho}=U D^{1 / 2} U^{-1}$. This is indeed feasible as shown in [1], but even for simple $2 \times 2$-matrices it involves relatively lengthy expressions and requires specific choices for the constants in order to guarantee that the eigenvalues are all real. This is also the case for the model considered here as seen from the determinant of $\rho$

$$
\operatorname{det} \rho=\frac{1-\gamma}{1+\gamma} b_{4}^{2}-b_{1}^{2}-b_{2}^{2}-b_{3}^{2}
$$

Evidently this expression might become negative, so that at least one of the two eigenvalues of $\rho$ would be negative and even for choices for which $\operatorname{det} \rho>0$ we may have two negative eigenvalues. We will not carry out this step here, but instead follow an easier way to find $\eta$ from (5) and compare thereafter with the solution (14).

\subsection{Solutions of the time-dependent Dyson relation}

In order to solve the time-dependent Dyson relation we need to make some pre-assumptions about the Hermitian Hamiltonian $h(t)$ and the map $\eta(t)$. We take $h(t)$ to be of the form as specified in (9) with $S_{z}=\sigma_{z}$ and assume $\eta(t)$ to be of the most generic Hermitian form

$$
\eta(t)=\left(\begin{array}{cc}
\eta_{1}(t) & \eta_{2}(t)-i \eta_{3}(t) \\
\eta_{2}(t)+i \eta_{3}(t) & \eta_{4}(t)
\end{array}\right) .
$$


Taking this Ansatz into (5) leads to seven different constraining equations:

$$
\begin{aligned}
\dot{\eta}_{1} & =\eta_{2} \frac{\gamma+1}{2}, \quad \dot{\eta}_{2}=\eta_{1} \frac{\gamma-1}{2}+\eta_{3} \frac{\chi}{2}=\eta_{4} \frac{\gamma+1}{2}+\eta_{3} \frac{\chi}{2}, \quad \dot{\eta}_{3}=\eta_{2} \frac{\chi}{2}, \quad \dot{\eta}_{4}=\eta_{2} \frac{\gamma-1}{2}, \\
\eta_{3}(\gamma+1)-\chi \eta_{1} & =\eta_{3}(1-\gamma)+\chi \eta_{4}=0
\end{aligned}
$$

Even though this system of equations is overdetermined, it can be solved by

$$
\eta_{1}(t)=\frac{c_{1}(\gamma+1)}{\chi^{1 / 2}(\gamma-1)}, \quad \eta_{2}(t)=\frac{c_{1} \dot{\chi}}{\chi^{3 / 2}(\gamma-1)}, \quad \eta_{3}(t)=\frac{c_{1} \chi^{1 / 2}}{\gamma-1}, \quad \eta_{4}(t)=\frac{c_{1}}{\chi^{1 / 2}},
$$

with one integration constant $c_{1} \in \mathbb{R}$ provided that the function $\chi$ satisfies the second-order non-linear differential equation

$$
\ddot{\chi}-\frac{3}{2} \frac{\dot{\chi}^{2}}{\chi}-\frac{1}{2} \phi^{2} \chi+\frac{1}{2} \chi^{3}=0 .
$$

Using the variable transformation $\chi=2 / \sigma^{2}$ this equation is converted into the Ermakov-Pinney equation [16,17]

$$
\ddot{\sigma}+\frac{1}{4} \phi^{2} \sigma=\frac{1}{\sigma^{3}}
$$

which is ubiquitous in the context of the TDSE, e.g. [18-23], and also some quantization schemes [24,25]. The general solution to this equation is known to be

$$
\sigma(t)=\left[A \sin ^{2}(\phi t / 2)+B \cos ^{2}(\phi t / 2) \pm 2 C \sin (\phi t / 2) \cos (\phi t / 2)\right]^{1 / 2},
$$

where the constants $A, B$ and $C$ are constraint as $A B-C^{2}=4 / \phi^{2}$, see [26]. Transforming back to $\chi$ and introducing the new real constants $c_{2}$ and $c_{3}$ via the relations $A=2\left(-c_{3} \pm \sqrt{1+c_{2}^{2}+c_{3}^{2}}\right) / \phi$ and $B=2\left(c_{3} \pm \sqrt{1+c_{2}^{2}+c_{3}^{2}}\right) / \phi$, we obtain the general solution to (19) in the form

$$
\chi(t)=\frac{\phi}{c_{2} \sin (\phi t)+c_{3} \cos (\phi t) \pm \sqrt{1+c_{2}^{2}+c_{3}^{2}}} .
$$

Thus with (18) and (16) we have obtained a generic solution for $\eta$.

Let us now compare this with the solution of the time-dependent quasi-Hermiticity relation obtained in the previous subsection. Computing $\eta^{2}$ from the above expressions and identifying the result as $\rho$ we can compare with the solution (14) obtained previously. Matching the constants as

$$
b_{1}=-\frac{2 c_{3} \gamma c_{1}^{2}}{(1-\gamma)^{2}}, \quad b_{2}=\frac{2 c_{2} \gamma c_{1}^{2}}{(1-\gamma)^{2}}, \quad b_{3}=\frac{2 \gamma c_{1}^{2}}{(1-\gamma)^{2}}, \quad b_{4}=\frac{2 \phi c_{1}^{2} \sqrt{1+c_{2}^{2}+c_{3}^{2}}}{(1-\gamma)^{3}}
$$

the two solutions become identical. Evidently these constants could not have been guessed in the approach of the previous subsection. With these values the determinant becomes

$$
\operatorname{det} \rho=\frac{4(1+\gamma) c_{1}^{4}}{(1-\gamma)^{3}}\left(1+c_{2}^{2}+c_{3}^{2}\right)
$$

which is positive for $|\gamma| \leq 1$. From the above it is clear that it is far easier to solve (5) directly, as it can essentially be reduced to some algebraic manipulations, a simple integration and the Ermakov-Pinney equation for which the general solution is known.

We have now obtained all the ingredients to compute the solution to the TDSE for the Hermitian system from (3). Assembling our results we obtain from $\phi_{ \pm}(t)=\sqrt{\mathcal{N}_{ \pm}} \eta(t) \Psi_{ \pm}(t)$ the normalized eigenvectors

$$
\phi_{ \pm}(t)=c_{1} \sqrt{\mathcal{N}_{ \pm} \chi}\left(\begin{array}{c}
\frac{1+\gamma}{\phi} i\left[e^{ \pm i t\left(E_{+}-E_{-}\right)}\left(i c_{2} \mp c_{3}\right)+1 \mp \sqrt{1+c_{2}^{2}+c_{3}^{2}}\right] \\
e^{ \pm i t\left(E_{+}-E_{-}\right)}\left( \pm i c_{2}+c_{3}\right) \pm 1-\sqrt{1+c_{2}^{2}+c_{3}^{2}}
\end{array}\right) e^{-i t E_{ \pm}}
$$

with normalization factors

$$
\mathcal{N}_{ \pm}=\frac{1-\gamma}{\mp 4 c_{1}^{2} \phi\left(\gamma \mp \sqrt{1+c_{2}^{2}+c_{3}^{2}}\right)}
$$


Form this we compute the expectation values

$$
\begin{aligned}
& \mathcal{N}_{ \pm}\left\langle\Psi_{ \pm}(t) \mid \rho(t) \Psi_{ \pm}(t)\right\rangle=\left\langle\phi_{ \pm}(t) \mid \phi_{ \pm}(t)\right\rangle=1 \\
& \mathcal{N}_{ \pm}\left\langle\Psi_{ \pm}(t) \mid \rho(t) \Psi_{\mp}(t)\right\rangle=\left\langle\phi_{ \pm}(t) \mid \phi_{\mp}(t)\right\rangle=\frac{\gamma\left( \pm c_{3}+i c_{2}\right)}{\sqrt{\phi^{2}+c_{2}^{2}+c_{3}^{2}}},
\end{aligned}
$$

which confirm that the time-evolution is indeed unitary. We also confirm the validity of the relation for the energy expectations (7) by computing

$$
\left\langle\phi_{ \pm}(t) \mid h(t) \phi_{ \pm}(t)\right\rangle=\mathcal{N}_{ \pm}\left\langle\Psi_{ \pm}(t) \mid \rho(t) \tilde{H}(t) \Psi_{ \pm}(t)\right\rangle=\frac{ \pm \phi^{2} \sqrt{1+c_{2}^{2}+c_{3}^{2}}-c_{2}^{2}-c_{3}^{2}}{2\left(\phi^{2}+c_{2}^{2}+c_{3}^{2}\right)} \chi(t)-\frac{\omega}{2} .
$$

While we found some explicit solutions, this example also demonstrates that one can not map to any arbitrary given target Hamiltonian, as $\chi(t)$ is restricted by the non-linear equation (19).

\section{A solvable equivalence pair of spin-1 models}

Increasing the dimension of the spin representation poses a more difficult challenge, but as we will see many of the features we observed for the spin-(1/2) model will survive. Let us next consider a generalization of the previous model to a spin 1 model where the matrices $S_{j}^{x}, S_{j}^{y}, S_{j}^{z}$ in (8) are taken to be the standard $3 \times 3$ spin- 1 matrices

$$
S^{x}=\frac{1}{\sqrt{2}}\left(\begin{array}{lll}
0 & 1 & 0 \\
1 & 0 & 1 \\
0 & 1 & 0
\end{array}\right), \quad S^{y}=\frac{1}{\sqrt{2}}\left(\begin{array}{ccc}
0 & -i & 0 \\
i & 0 & -i \\
0 & i & 0
\end{array}\right), \quad S^{z}=\left(\begin{array}{ccc}
1 & 0 & 0 \\
0 & 0 & 0 \\
0 & 0 & -1
\end{array}\right) \text {. }
$$

Choosing the constants $c_{x}, c_{y}, c_{\omega}$ conveniently this Hamiltonian simplifies for $N=1$ to

$$
H_{1}^{1}=-\frac{1}{\sqrt{2}}\left(S^{y}+\frac{\omega}{\sqrt{2}} \mathbb{I}+i \gamma S^{x}\right)=-\frac{1}{2}\left(\begin{array}{ccc}
\omega & i(\gamma-1) & 0 \\
i(\gamma+1) & \omega & i(\gamma-1) \\
0 & i(\gamma+1) & \omega
\end{array}\right) .
$$

The corresponding TDSE (1) is solved by

$$
\Psi_{k}(t)=\left(\begin{array}{c}
(-1)^{k}(1-\gamma) \\
2 i k \tilde{\phi} \\
1-\gamma
\end{array}\right) e^{-i t E_{k}}, \quad E_{k}=-\frac{\omega}{2}+k \tilde{\phi}, \quad k=0, \pm 1,
$$

where $\tilde{\phi}:=\sqrt{\left(1-\gamma^{2}\right) / 2}$. Once again in the parameter region $|\gamma| \leq 1$ the non-Hermitian Hamiltonian (31) possesses a real eigenvalue spectrum. Next we solve (4) and (5).

\subsection{Solutions of the time-dependent quasi-Hermiticity relation}

Assuming the time-dependent metric operator to be Hermitian we substitute the most generic Ansatz

$$
\rho(t)=\left(\begin{array}{ccc}
\rho_{1}(t) & \rho_{2}(t)-i \rho_{3}(t) & \rho_{4}(t)-i \rho_{5}(t) \\
\rho_{2}(t)+i \rho_{3}(t) & \rho_{6}(t) & \rho_{7}(t)-i \rho_{8}(t) \\
\rho_{4}(t)+i \rho_{5}(t) & \rho_{7}(t)+i \rho_{8}(t) & \rho_{9}(t)
\end{array}\right)
$$

into the time-dependent quasi-Hermiticity relation (4) obtaining in principle 18 equations for the nine real functions $\rho_{i}(t), i=1, \ldots, 9$. Excluding vanishing and related ones we are left with nine equations

$$
\begin{array}{ll}
\dot{\rho}_{1}=\rho_{2}(\gamma+1), \quad \dot{\rho}_{2}=\rho_{1} \frac{\gamma-1}{2}+\left(\rho_{4}+\rho_{6}\right) \frac{\gamma+1}{2}, & \dot{\rho}_{3}=\rho_{5} \frac{\gamma+1}{2}, \\
\dot{\rho}_{4}=\rho_{2} \frac{\gamma-1}{2}+\rho_{7} \frac{\gamma+1}{2}, \quad \dot{\rho}_{5}=\rho \frac{\gamma-1}{2}+\rho_{8} \frac{\gamma+1}{2}, & \dot{\rho}_{6}=\rho_{2}(\gamma-1)+\rho_{7}(\gamma+1), \\
\dot{\rho}_{7}=\left(\rho_{4}+\rho_{6}\right) \frac{\gamma-1}{2}+\rho_{9} \frac{\gamma+1}{2}, \quad \dot{\rho}_{8}=\rho_{5} \frac{\gamma-1}{2}, & \dot{\rho}_{9}=\rho_{7}(\gamma-1) .
\end{array}
$$


Once again as in the spin-(1/2) case we have as many equations as unknown functions and it is straightforward to solve these equations, as substitutions lead to simple integrals. We find the solutions

$$
\begin{aligned}
& \rho_{1}(t)=\frac{\left(2 b_{4}+3 b_{5}\right)(\gamma+1)}{8(1-\gamma)}+\tilde{\Gamma}_{b_{6}}^{b_{7}}+\breve{\Gamma}_{b_{8}}^{b_{9}}, \quad \rho_{2}(t)=\frac{\phi}{1+\gamma}\left[\tilde{\Gamma}_{-b_{7}}^{b_{6}}+2 \breve{\Gamma}_{-b_{9}}^{b_{8}}\right], \\
& \rho_{3}(t)=\frac{1+\gamma}{2 \phi}\left[\tilde{\Gamma}_{b_{2}}^{-b_{1}}\right]+b_{3}, \quad \rho_{4}(t)=\frac{\gamma-1}{\gamma+1} \breve{\Gamma}_{b_{8}}^{b_{9}}+\frac{1}{8}\left(6 b_{4}+b_{5}\right), \quad \rho_{5}(t)=\tilde{\Gamma}_{b_{1}}^{b_{2}}, \\
& \rho_{6}(t)=2 \frac{\gamma-1}{\gamma+1} \breve{\Gamma}_{b_{8}}^{b_{9}}-\frac{1}{4}\left(2 b_{4}-b_{5}\right), \quad \rho_{7}(t)=\frac{1}{\sqrt{2}}\left(\frac{1-\gamma}{1+\gamma}\right)^{3 / 2}\left[\tilde{\Gamma}_{-b_{7}}^{b_{6}}+2 \breve{\Gamma}_{b_{9}}^{-b_{8}}\right], \\
& \rho_{8}(t)=\frac{\phi}{1+\gamma} \tilde{\Gamma}_{-b_{2}}^{b_{1}}+\frac{1-\gamma}{1+\gamma} b_{3}, \quad \rho_{9}(t)=\left(\frac{1-\gamma}{1+\gamma}\right)^{2}\left[-\tilde{\Gamma}_{b_{6}}^{b_{7}}+\breve{\Gamma}_{b_{8}}^{b_{9}}\right]+\frac{1-\gamma}{8(1+\gamma)}\left(2 b_{4}+3 b_{5}\right),
\end{aligned}
$$

with nine integration constants $b_{i}, i=1, \ldots, 9$. We abbreviated $\tilde{\Gamma}_{x}^{y}:=x \sin (\tilde{\phi} t)+y \cos (\tilde{\phi} t)$ and $\breve{\Gamma_{x}^{y}}:=x \sin (2 \tilde{\phi} t)+$ $y \cos (2 \tilde{\phi} t)$. For this solution it is even less evident to chose suitable constants and simplifying choices by setting some of the $b_{i}$ to zero usually yield negative eigenvalues for $\rho$. Thus we will not compute the root, but return to this solution below for comparison.

\subsection{Solutions of the time-dependent Dyson relation}

Instead we solve the time-dependent Dyson equation (5). We assume a similar form for our Hermitian target Hamiltonian as in (9) and take $S^{z}$ to be a spin-1 matrix, denote $\chi=X$ and take $\eta(t)$ to be of the Hermitian form

$$
\eta(t)=\left(\begin{array}{ccc}
\eta_{1}(t) & \eta_{2}(t)-i \eta_{3}(t) & \eta_{4}(t)-i \eta_{5}(t) \\
\eta_{2}(t)+i \eta_{3}(t) & \eta_{6}(t) & \eta_{7}(t)-i \eta_{8}(t) \\
\eta_{4}(t)+i \eta_{5}(t) & \eta_{7}(t)+i \eta_{8}(t) & \eta_{9}(t)
\end{array}\right)
$$

Substituting these expressions into the time-dependent Dyson equation (5) yields in principle 18 equations for the real functions $\eta_{i}(t), i=1, \ldots, 9$. We obtain

$$
\begin{aligned}
& \dot{\eta}_{1}=\eta_{2} \frac{\gamma+1}{2}, \quad \dot{\eta}_{2}=\eta_{1} \frac{\gamma-1}{2}+\eta_{3} \frac{X}{2}+\eta_{4} \frac{\gamma+1}{2}=\eta_{6} \frac{\gamma+1}{2}, \quad \dot{\eta}_{3}=-\eta_{2} \frac{X}{2}+\eta_{5} \frac{\gamma+1}{2}=0, \\
& \dot{\eta}_{4}=\eta_{2} \frac{\gamma-1}{2}+\eta_{5} \frac{X}{2}=\eta_{5} \frac{X}{2}+\eta_{7} \frac{\gamma+1}{2}, \quad \dot{\eta}_{5}=\eta_{3} \frac{\gamma-1}{2}-\eta_{4} \frac{X}{2}=\eta_{4} \frac{X}{2}-\eta_{8} \frac{\gamma+1}{2}, \\
& \dot{\eta}_{6}=\eta_{2} \frac{\gamma-1}{2}+\eta_{7} \frac{\gamma+1}{2}, \quad \dot{\eta}_{7}=\eta_{4} \frac{\gamma-1}{2}+\eta_{8} \frac{X}{2}+\eta_{9} \frac{\gamma+1}{2}=\eta_{6} \frac{\gamma-1}{2}, \\
& \dot{\eta}_{8}=\eta_{5} \frac{\gamma-1}{2}-\eta_{7} \frac{X}{2}=0, \quad \dot{\eta}_{9}=\eta_{7} \frac{\gamma-1}{2}
\end{aligned}
$$

and

$$
(1+\gamma) \eta_{3}-X \eta_{1}=(1-\gamma) \eta_{3}+(1+\gamma) \eta_{8}=(1-\gamma) \eta_{8}+X \eta_{9}=0
$$

Unlike the system of equations for the metric operator this set is overdetermined. Nonetheless, they may be solved by

$$
\begin{array}{ll}
\eta_{1}(t)=\frac{c_{1}}{X}, \quad \eta_{2}(t)=-\frac{2 c_{1} \dot{X}}{(1+\gamma)^{2} X^{2}}, \quad \eta_{3}(t)=\frac{c_{1}}{1+\gamma}, & \eta_{4}(t)=\frac{c_{1}\left(4 \dot{X}^{2}-X^{4}\right)}{2(1+\gamma)^{2} X^{3}}, \\
\eta_{5}(t)=-\frac{2 c_{1} \dot{X}}{(1+\gamma)^{2} X}, \quad \eta_{6}(t)=\frac{c_{1}\left(4 \dot{X}^{2}+X^{4}-4 \phi^{2} X^{2}\right)}{2(1+\gamma)^{2} X^{3}}, & \eta_{7}(t)=\frac{2(1-\gamma) c_{1} \dot{X}}{(1+\gamma)^{2} X^{2}}, \\
\eta_{8}(t)=\frac{c_{1}(\gamma-1)}{(\gamma+1)^{2}}, \quad \eta_{9}(t)=\frac{c_{1}(1-\gamma)^{2}}{(1+\gamma)^{2} X}, &
\end{array}
$$

where $X(t)$ is restricted to obey the second-order non-linear differential equation

$$
\ddot{X}-\frac{3}{2} \frac{\dot{X}^{2}}{X}-\frac{1}{2} \tilde{\phi}^{2} X+\frac{X^{3}}{8}=0 .
$$


This equation closely resembles (19) and we can once more transform it to the Ermakov-Pinney equation (20) with $\sigma \rightarrow \tilde{\sigma}, \phi \rightarrow \tilde{\phi}$ by using $X=4 / \tilde{\sigma}^{2}$ in this case. Following the same steps of the previous subsection we obtain the general solution for (40) as

$$
X(t)=\frac{2 \tilde{\phi}}{c_{2} \sin (\tilde{\phi} t)+c_{3} \cos (\tilde{\phi} t) \pm \sqrt{1+c_{2}^{2}+c_{3}^{2}}} .
$$

Again we compute $\eta^{2}$ and compare the result with $\rho$ from the previous subsection. Identifying the constants as

$$
\begin{aligned}
& b_{1}=-\frac{4 \gamma^{2} c_{1}^{2} c_{3}}{(1+\gamma)^{4}}, \quad b_{2}=\frac{4 \gamma^{2} c_{1}^{2} c_{2}}{(1+\gamma)^{4}}, \quad b_{3}=\frac{2 \gamma c_{1}^{2} \sqrt{1+c_{2}^{2}+c_{3}^{2}}}{\phi(1+\gamma)^{3}}, \quad b_{4}=\frac{2 c_{1}^{2}\left[\phi^{2}\left(3-c_{2}^{2}-c_{3}^{2}\right)-2\right]}{(1+\gamma)^{4}}, \\
& b_{5}=\frac{2 c_{1}^{2}\left(3+\gamma^{2}\right)\left(1+c_{2}^{2}+c_{3}^{2}\right)}{(1+\gamma)^{4}}, \quad b_{6}=\frac{2 \gamma c_{1}^{2} c_{2} \sqrt{1+c_{2}^{2}+c_{3}^{2}}}{\phi^{2}(1+\gamma)^{2}}, \quad b_{7}=\frac{2 \gamma c_{1}^{2} c_{3} \sqrt{1+c_{2}^{2}+c_{3}^{2}}}{\phi^{2}(1+\gamma)^{2}}, \\
& b_{8}=\frac{\gamma^{2} c_{1}^{2} c_{2} c_{3}}{\phi^{2}(1+\gamma)^{2}}, \quad b_{9}=\frac{\gamma^{2} c_{1}^{2}\left(c_{2}^{2}-c_{3}^{2}\right)}{2 \phi^{2}(1+\gamma)^{2}},
\end{aligned}
$$

the two solutions coincide. This demonstrates once more why simple choices for the constants $c_{i}$ did not yield meaningful solutions for $\eta$. Using the values (42) we compute the determinant

$$
\operatorname{det} \rho=8 \frac{(1-\gamma)^{3}}{(1+\gamma)^{9}} c_{1}^{6}\left(1+c_{2}^{2}+c_{3}^{2}\right)^{3},
$$

which is positive for the parameter range of interest.

Having computed the Dyson map and the solution to the TDSE for $H$ we obtain the solution for the TDSE involving $h(t)$ from $(3)$

$$
\begin{aligned}
& \phi_{ \pm}(t)=\left(\begin{array}{c}
-X(t)\left[1 \mp \sqrt{1+c_{2}^{2}+c_{3}^{2}}+e^{i t\left(E_{ \pm}-E_{0}\right)}\left(i c_{2} \mp c_{3}\right)[1 \mp 2 \phi / X(t)]\right] \\
2(1-\gamma)\left(-c_{2} \mp i c_{3}\right) e^{i t\left(E_{ \pm}-E_{0}\right)} \\
(1-\gamma) X(t)\left[1 \pm \sqrt{1+c_{2}^{2}+c_{3}^{2}}-e^{i t\left(E_{ \pm}-E_{0}\right)}\left(i c_{2} \mp c_{3}\right)[1 \pm 2 \phi / X(t)]\right]
\end{array}\right) \frac{c_{1} e^{-i t E_{ \pm}}}{1+\gamma}, \\
& \phi_{0}(t)=\left(\begin{array}{c}
X(t)\left[i c_{3} \sin (\phi t)-i c_{2} \cos (\phi t)-1\right]+2 \phi \sqrt{1+c_{2}^{2}+c_{3}^{2}} \\
2 i(1-\gamma) \\
(1-\gamma)\left[X(t)\left[-i c_{3} \sin (\phi t)+i c_{2} \cos (\phi t)-1\right]+2 \phi \sqrt{1+c_{2}^{2}+c_{3}^{2}}\right]
\end{array}\right) \frac{c_{1} e^{-i t E_{0}}}{1+\gamma} .
\end{aligned}
$$

As in the previous section we can use these expressions to confirm that the time-evolution is unitary and also verify (7).

\section{A solvable equivalence pair of spin-(3/2) models}

Finally we also consider a spin-(3/2) model and take the matrices $S_{j}^{x}, S_{j}^{y}, S_{j}^{z}$ in (8) to be $4 \times 4$ spin-(3/2) matrices

$$
S^{x}=\frac{1}{2}\left(\begin{array}{cccc}
0 & \sqrt{3} & 0 & 0 \\
\sqrt{3} & 0 & 2 & 0 \\
0 & 2 & 0 & \sqrt{3} \\
0 & 0 & \sqrt{3} & 0
\end{array}\right), \quad S^{y}=\frac{i}{2}\left(\begin{array}{cccc}
0 & -\sqrt{3} & 0 & 0 \\
\sqrt{3} & 0 & -2 & 0 \\
0 & 2 & 0 & -\sqrt{3} \\
0 & 0 & \sqrt{3} & 0
\end{array}\right), \quad S^{z}=\frac{1}{2}\left(\begin{array}{cccc}
3 & 0 & 0 & 0 \\
0 & 1 & 0 & 0 \\
0 & 0 & -1 & 0 \\
0 & 0 & 0 & -3
\end{array}\right),
$$

at site $j$. Choosing the constants $c_{x}, c_{y}, c_{\omega}$ conveniently, for $N=1$ this Hamiltonian simplifies to

$$
H_{1}^{3 / 2}=-\frac{1}{6}\left(S^{y}+\frac{2 \omega}{3} \mathbb{I}+i \gamma S^{x}\right)=-\frac{1}{4}\left(\begin{array}{ccccc}
\omega & i \frac{\gamma-1}{\sqrt{3}} & 0 & 0 \\
i \frac{\gamma-1}{\sqrt{3}} & \omega & i \frac{\gamma-1}{6} & 0 \\
0 & i \frac{\gamma-1}{6} & \omega & \frac{\gamma-1}{\sqrt{3}} \\
0 & 0 & \frac{\gamma-1}{\sqrt{3}} & \omega
\end{array}\right) .
$$


The corresponding TDSE (1) is solved to

$$
\Psi_{k}(t)=\left(\begin{array}{c}
i(1-\gamma)^{3 / 2} \\
-2 \sqrt{3} k \hat{\phi}(1-\gamma)^{1 / 2} \\
2 i \sqrt{3}\left(k^{2}-2|k|\right) \hat{\phi}(1+\gamma)^{1 / 2} \\
\operatorname{sign}(k)(|k|-2)(1+\gamma)^{3 / 2}
\end{array}\right) e^{-i t E_{k}}, \quad E_{k}=-\frac{1}{2} k \hat{\phi}-\frac{\omega}{4}, \quad k= \pm 1, \pm 3
$$

where $\hat{\phi}:=\sqrt{1-\gamma^{2}} / 6$. The eigenvalue spectrum is real for the same parameter range as in the previous subsections.

Here we will only solve the time-dependent Dyson equation (5) to see whether the features of the spin-(1/2) and spin-1 models are also present in this model. We assume a similar form for our Hermitian target Hamiltonian as in (9), denote $\chi=\Xi$ and take $\eta(t)$ to be of the Hermitian form

$$
\eta(t)=\left(\begin{array}{cccc}
\eta_{1}(t) & \eta_{2}(t)-i \eta_{3}(t) & \eta_{4}(t)-i \eta_{5}(t) & \eta_{6}(t)-i \eta_{7}(t) \\
\eta_{2}(t)+i \eta_{3}(t) & \eta_{8}(t) & \eta_{9}(t)-i \eta_{10}(t) & \eta_{11}(t)-i \eta_{12}(t) \\
\eta_{4}(t)+i \eta_{5}(t) & \eta_{7}(t)+i \eta_{8}(t) & \eta_{13}(t) & \eta_{14}(t)-i \eta_{15}(t) \\
\eta_{6}(t)+i \eta_{7}(t) & \eta_{11}(t)+i \eta_{12}(t) & \eta_{14}(t)+i \eta_{15}(t) & \eta_{16}(t)
\end{array}\right)
$$

Substituting these expressions into the time-dependent Dyson equation (5) yields in principle 32 equations for the $\eta_{i}(t), i=1, \ldots, 16$. Once again the system is highly overdetermined, but remarkably it can be solved similarly as in the previous sections. Here we only present the solutions to these equations. We find

$$
\begin{aligned}
\eta_{1}(t) & =\frac{c_{1}}{\Xi^{3 / 2}}, \quad \eta_{2}(t)=-\frac{6 \sqrt{3} c_{1} \dot{X}}{(1+\gamma) \Xi^{5 / 2}}, \quad \eta_{3}(t)=\frac{3 \sqrt{3} c_{1}}{(1+\gamma) \Xi^{1 / 2}}, \quad \eta_{4}(t)=\frac{9 \sqrt{3} c_{1}\left(4 \dot{\Xi}^{2}-\Xi^{4}\right)}{(1+\gamma)^{2} \Xi^{7 / 2}} \\
\eta_{5}(t) & =-\frac{36 \sqrt{3} c_{1} \dot{\Xi}}{(1+\gamma)^{2} \Xi^{3 / 2}}, \quad \eta_{6}(t)=\frac{54 c_{1}\left(3 \dot{\Xi} \Xi^{4}-4 \dot{\Xi}^{3}\right)}{(1+\gamma)^{3} \Xi^{9 / 2}}, \quad \eta_{7}(t)=\frac{27 c_{1}\left(12 \dot{\Xi}^{2}-\Xi^{4}\right)}{(1+\gamma)^{3 / 2} \Xi^{5 / 2}} \\
\eta_{8}(t) & =\frac{6 c_{1}\left(12 \dot{\Xi}^{2}+3 \Xi^{4}-\hat{\phi}^{2} \Xi^{2}\right)}{(\gamma+1)^{2} \Xi^{7 / 2}}, \quad \eta_{9}(t)=\frac{18 c_{1} \dot{\Xi}\left(4 \hat{\phi}^{2} \Xi^{2}-12 \dot{\Xi}^{2}-3 \Xi^{4}\right)}{(\gamma+1)^{3} \Xi^{9 / 2}} \\
\eta_{10}(t) & =\frac{9 c_{1} \dot{\Xi}\left(4 \hat{\phi}^{2} \Xi^{2}-12 \dot{\Xi}^{2}-3 \Xi^{4}\right)}{(\gamma+1)^{3} \Xi^{5 / 2}}, \quad \eta_{11}(t)=\frac{9 \sqrt{3} c_{1}(1-\gamma)\left(\Xi^{4}-4 \dot{X}^{2}\right)}{(1+\gamma)^{3} \Xi^{7 / 2}} \\
\eta_{12}(t) & =\frac{36 \sqrt{3} c_{1}(1-\gamma) \dot{\Xi}}{(1+\gamma)^{3} \Xi^{3 / 2}}, \quad \eta_{13}(t)=\frac{6 c_{1}(\gamma-1)\left(12 \dot{\Xi}^{2}+3 \Xi^{4}-\hat{\phi}^{2} \Xi^{2}\right)}{(1+\gamma)^{3} \Xi^{7 / 2}}, \\
\eta_{14}(t) & =-\frac{6 \sqrt{3} c_{1}(1-\gamma)^{2} \dot{\Xi}}{(1+\gamma)^{3} \Xi^{5 / 2}}, \quad \eta_{15}(t)=\frac{3 \sqrt{3} c_{1}(1-\gamma)^{2}}{(1+\gamma)^{3} \Xi^{1 / 2}}, \quad \eta_{16}(t)=\frac{c_{1}(\gamma-1)^{3}}{(1+\gamma)^{3} \Xi^{3 / 2}}
\end{aligned}
$$

where $\Xi(t)$ has to obey the second-order non-linear differential equation

$$
\ddot{\Xi}-\frac{3}{2} \frac{\dot{\Xi}^{2}}{\Xi}-\frac{1}{2} \hat{\phi}^{2} \Xi+\frac{\Xi^{3}}{8}=0 .
$$

As in the previous subsection we can transform it to the Ermakov-Pinney equation (20) with $\sigma \rightarrow \hat{\sigma}, \phi \rightarrow \hat{\phi}$ using $\Xi=4 / \hat{\sigma}^{2}$ in this case and therefore we have

$$
\Xi(t)=\frac{2 \hat{\phi}}{c_{2} \sin (\hat{\phi} t)+c_{3} \cos (\hat{\phi} t) \pm \sqrt{1+c_{2}^{2}+c_{3}^{2}}} .
$$

Computing from this $\rho=\eta^{2}$, we evaluate the determinant to

$$
\operatorname{det} \rho=\frac{6^{6}(1-\gamma)^{6} c_{1}^{8}}{(1+\gamma)^{18}}\left(1+c_{2}^{2}+c_{3}^{2}\right)^{6}
$$

which is always positive for the parameter range of interest. Naturally (3) yields once more the soltution to the TDSE for $h(t)$. 


\section{Conclusions}

We have demonstrated that metric representations lead to consistent descriptions equivalent to the operator representation by providing further solutions to the time-dependent quasi-Hermiticity relation (4) and the time-dependent Dyson relation (5). For the spin models we considered here we observed that the determining relation for the metric operator (4) converts into as many equations as unknown functions. The equations are easily decoupled and integrated to determine the metric operator. However, the diagonalization needed in order to take the square root is usual and moreover requires specific choices for the constants involved to ensure that all eigenvalues are positive. As we have demonstrated simple choices are usually not evident or do not even exist. In order to bypass this step we pursued what turned out to be an easier approach and solved the time-dependent Dyson relation (5) instead. Assuming a general form for the Hermitian Hamiltonian in (5) converts it into an overdetermined set of equations for the components of the Dyson map. Remarkably these equations can be decoupled and solved by simple integrations for the components of $\eta$. The time-dependent equation occurring in the Hermitian Hamiltonian is restricted by a non-linear equation that can be converted into the Ermakov-Pinney equation. This feature was observed in all three spin models considered here and based on this observation we conjecture that it might be universal and will hold for all higher-spin representations.

Evidently there are many interesting open problems left for future research, such as a more extensive treatment of systems with explicitly time-dependent non-Hermitian Hamiltonian and with regard to the spin models more sites pose a natural challenge.

TF is supported by a City, University of London Research Fellowship.

Open Access This is an open access article distributed under the terms of the Creative Commons Attribution License (http://creativecommons.org/licenses/by/4.0), which permits unrestricted use, distribution, and reproduction in any medium, provided the original work is properly cited.

\section{References}

1. A. Fring, T. Frith, Phys. Rev. A 95, 010102(R) (2017).

2. C.M. Bender, S. Boettcher, Phys. Rev. Lett. 80, 5243 (1998).

3. C.M. Bender, Rep. Prog. Phys. 70, 947 (2007).

4. A. Mostafazadeh, Int. J. Geom. Methods Mod. Phys. 7, 1191 (2010).

5. F. Cannata, M. Ioffe, G. Junker, D.J. Nishnianidze, J. Phys. A 32, 3583 (1999).

6. A.A. Andrianov, F. Cannata, J. Phys. A 37, 10297 (2004).

7. C. Figueira de Morisson Faria, A. Fring, J. Phys. A 39, 9269 (2006).

8. C. Figueira de Morisson Faria, A. Fring, Laser Phys. 17, 424 (2007).

9. A. Mostafazadeh, Phys. Lett. B 650, 208 (2007).

10. M. Znojil, Phys. Rev. D 78, 085003 (2008).

11. A. Fring, M.H.Y. Moussa, Phys. Rev. A 93, 042114 (2016).

12. F.S. Luiz, M.A. Pontes, M.H.Y. Moussa, Unitarity of the time-evolution and observability of non-Hermitian Hamiltonians for time-dependent Dyson maps, arXiv:1611.08286 (2016).

13. A. Fring, M.M.H.Y. Moussa, Phys. Rev. A 94, 042128 (2016).

14. G. von Gehlen, J. Phys. A 24, 5371 (1991).

15. O.A. Castro-Alvaredo, A. Fring, J. Phys. A 42, 465211 (2009).

16. V. Ermakov, Univ. Izv. Kiev. 20, 1 (1880).

17. E. Pinney, Proc. Am. Math. Soc. 1, 681 (1950).

18. A. Hone, Phys. Lett. A 263, 347 (1999).

19. R.M. Hawkins, J.E. Lidsey, Phys. Rev. D 66, 023523 (2002).

20. J.R. Choi, B.H. Kweon, Int. J. Mod. Phys. B 16, 4733 (2002).

21. J.R. Choi, Int. J. Theor. Phys. 42, 853 (2003).

22. N. Ferkous, A. Bounames, M. Maamache, Phys. Scr. 88, 35001 (2013).

23. S. Dey, A. Fring, Phys. Rev. D 90, 084005 (2014).

24. M.V. Ioffe, H. Korsch, Phys. Lett. A 311, 200 (2003).

25. S. Dey, A. Fring, L. Gouba, J. Phys. A 48, 40FT01 (2015).

26. C. Eliezer, A. Gray, SIAM J. Appl. Math. 30, 463 (1976). 